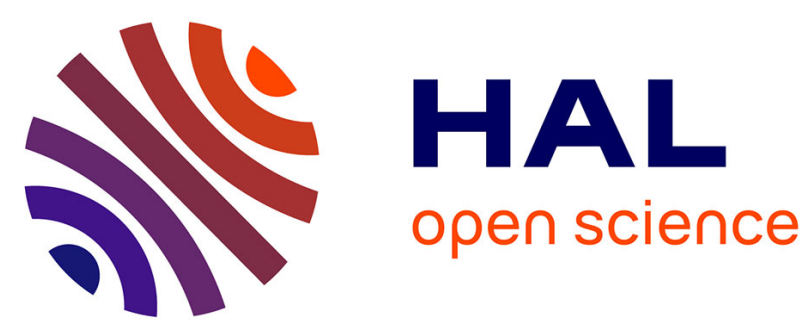

\title{
Low-frequency fluctuations in an external-cavity laser leading to extreme events
}

\author{
Daeyoung Choi, Michael J Wishon, J. Barnoud, C. y Chang, Y. Bouazizi, A. \\ Locquet, D. S Citrin
}

\section{To cite this version:}

Daeyoung Choi, Michael J Wishon, J. Barnoud, C. y Chang, Y. Bouazizi, et al.. Low-frequency fluctuations in an external-cavity laser leading to extreme events. Physical Review E , 2016, 93 (4), 10.1103/PhysRevE.93.042216 . hal-03048499

\section{HAL Id: hal-03048499 \\ https://hal.science/hal-03048499}

Submitted on 9 Dec 2020

HAL is a multi-disciplinary open access archive for the deposit and dissemination of scientific research documents, whether they are published or not. The documents may come from teaching and research institutions in France or abroad, or from public or private research centers.
L'archive ouverte pluridisciplinaire HAL, est destinée au dépôt et à la diffusion de documents scientifiques de niveau recherche, publiés ou non, émanant des établissements d'enseignement et de recherche français ou étrangers, des laboratoires publics ou privés. 


\title{
Low-frequency fluctuations in an external-cavity laser leading to extreme events
}

\author{
Daeyoung Choi, ${ }^{1,2}$ Michael J. Wishon, ${ }^{1,2}$ J. Barnoud ${ }^{1,2}$ C. Y. Chang, ${ }^{1,3}$ Y. Bouazizi, ${ }^{1,2}$ A. Locquet,,${ }^{1,2}$ and D. S. Citrin ${ }^{1,2, *}$ \\ ${ }^{1}$ UMI 2958 Georgia Tech-CNRS, Georgia Tech Lorraine, 2 Rue Marconi F-57070, Metz, France \\ ${ }^{2}$ School of Electrical and Computer Engineering, Georgia Institute of Technology, Atlanta, Georgia 30332-0250, USA \\ ${ }^{3}$ School of Physics, Georgia Institute of Technology, Atlanta, Georgia 30332-0430, USA \\ (Received 29 January 2016; revised manuscript received 30 March 2016; published 22 April 2016)
}

\begin{abstract}
We experimentally investigate the dynamical regimes of a laser diode subject to external optical feedback in light of extreme-event (EE) analysis. We observe EEs in the low-frequency fluctuations (LFFs) regime. This number decreases to negligible values when the laser transitions towards fully developed coherence collapse as the injection current is increased. Moreover, we show that EEs observed in the LFF regime are linked to high-frequency pulsing events observed after a power dropout. Finally, we prove experimentally that the observation of EEs in the LFF regimes is robust to changes in operational parameters.
\end{abstract}

DOI: 10.1103/PhysRevE.93.042216

\section{INTRODUCTION}

An extreme event (EE) is defined as a sudden surge of exceptional amplitude compared to the typical dynamics of a system. EEs were first reported in oceanography $[1,2]$ as very high amplitude waves, called rogue or freak waves, that appear unexpectedly, and that occur much more often than one would expect assuming a Gaussian or any other shorttailed distribution. EEs are not restricted to hydrodynamics; they have been observed in several other fields including economics [3], meteorology [4], acoustics [5], and optics [6-16].

Bonatto et al. [6] showed that rare giant pulses could be observed in a purely deterministic dynamical system composed of a semiconductor slave laser optically injected by a semiconductor master laser. Reference [15] demonstrated that these giant pulses could be predicted with a large anticipation time and originated in a crisis-like process that creates a narrow "rogue wave door" in phase space. Perrone et al. [16] further showed numerically that a weak modulation of the pump current could be used to control the probability of occurrence of these giant pulses, or even suppress them.

Laser diodes (LDs) subjected to optical feedback, also known as external cavity lasers (ECL), are of great intrinsic interest and have also led to a large number of applications [17]. In the short-cavity case, numerical simulations show that in certain parameter ranges, associated with an expansion of an attractor in phase space, occasional EEs can be observed [9]. In the special case of optical feedback provided by an external phase-conjugating mirror, extreme intensity pulses with statistical properties similar to those of rogue waves have been observed [8] and reproduced numerically [13] with a rate equation model.

EEs are determined by defining an EE threshold $E E_{\mathrm{th}}$ : features in a time series that exceed $E E_{\text {th }}$ are deemed EEs. One common criterion for determining the threshold uses the standard deviation $\sigma$ of the time series: any local maximum (or minimum) larger (or smaller) than the mean plus (or minus) 4 to 8 times $\sigma$ is considered an EE [1]. Another common criterion uses the abnormality index $\left(A_{I}\right)$ [2], which is the ratio between

\footnotetext{
*Corresponding author: david.citrin@ece.gatech.edu
}

the height of the wave and the average wave height among onethird of the highest waves in a time series. Any wave for which $A_{I}>2$ is considered an EE. We explored EEs determined by the $4 \sigma, 6 \sigma$, and $A_{I}$ criteria. We confirmed that regardless of criterion, the EE frequency exhibits the same trends with smooth transitions as a function of $J$ and only the scales are different. We chose to focus on the $+6 \sigma$ criterion as $E E_{\text {th }}$ since it gave us good statistics without requiring extremely long experimental time series. Also, we only considered maxima in determining the EEs as extreme minima are far less frequent.

\section{EXPERIMENTAL SETUP}

In this article, we observe experimentally the existence and frequency of EEs in the optical intensity $I(t)$ of a LD subjected to conventional, i.e., non-phase-conjugating, timedelayed optical feedback. The experimental setup is shown in Fig. 1; for more details, see [18]. The external cavity length $L$ corresponds to the time delay $\tau=2 L / c$ of the light with $c$ the in vacuo speed of light. A real-time oscilloscope with a $12 \mathrm{GHz}$ bandwidth is used to capture the intensity of the time series $I(t)$ at a rate of $40 \mathrm{GS} / \mathrm{s}$. The LD is a Mitsubishi ML925B11F. Its threshold current $J_{t h}$ is $8.9 \mathrm{~mA}$ without optical feedback, and its emission wavelength is $\sim 1550 \mathrm{~nm}$. A quarter-wave plate in the external cavity is rotated to control the feedback strength $\eta$; $\eta=1$ corresponds to the maximum feedback strength $(\sim 20 \%$ of the optical power reaching the emitting facet of the LD) that is fed back onto the LD. Depending on the feedback strength $\eta$, injection current $J$, and external cavity length $L$, the ECL exhibits various dynamical regimes $[19,20]$, the most relevant being low frequency fluctuation (LFF) and fully developed coherence collapse (CC) [17]. We varied the injection current from 8.0 to $18.0 \mathrm{~mA}$ by $0.1 \mathrm{~mA}$ increments, which allowed us to observe the transition from LFF to CC in detail, and record the corresponding optical intensity times series, each consisting of $3 \times 10^{6}$ samples.

\section{RESULTS AND DISCUSSION}

Of specific interest to this study is the transition between LFF and CC. LFF typically occurs near or slightly above $J_{\text {th }}$ and exhibits a succession of intervals of average power build-ups interrupted by abrupt power dropouts at apparently 


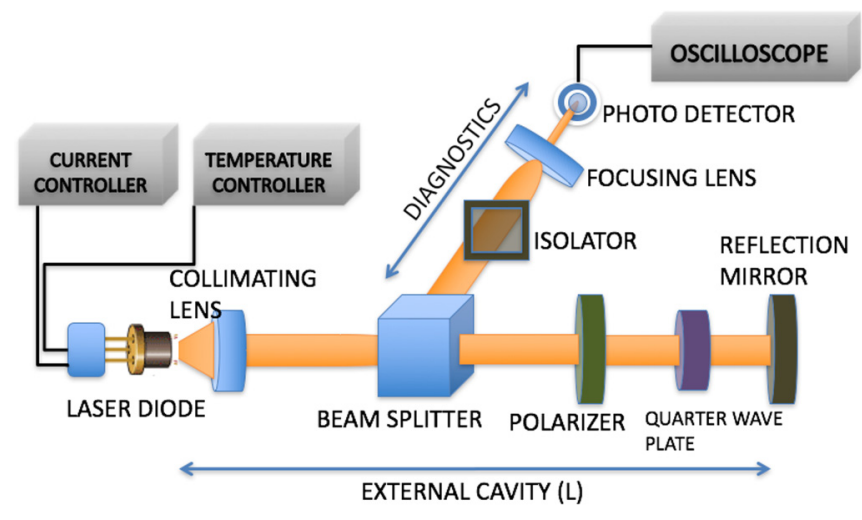

FIG. 1. Experimental setup. Light from the laser diode (LD) is split by a beam splitter. One optical path provides feedback, which is adjusted for feedback strength $\eta$ and external cavity length $L$. The other path is used for detection of the optical intensity $I(t)$.

randomly distributed times. The underlying instantaneous power is constituted by irregular intensity pulses whose duration is in the several tens to a few hundreds of ps [21]. LFF can be easily identified by the presence of a slow dynamics (several tens or a few hundreds of $\mathrm{MHz}$ ), corresponding to the alternation between buildups and dropouts, that is evident from a low-pass filtered time series and the rf spectrum. CC typically happens at larger $J$ and displays multi-GHz fluctuations of the optical intensity around its average value.

In Fig. 2 we plot examples of the standardized probability distribution function (pdf) of the intensity time series in (a) LFF and (b) CC. The standardized pdf is defined as $[I(t)-\langle I\rangle] / \sigma$ with $\langle I\rangle$ the time-averaged intensity. Note that the vertical scale is logarithmic. We observe that in LFF, the standardized pdf

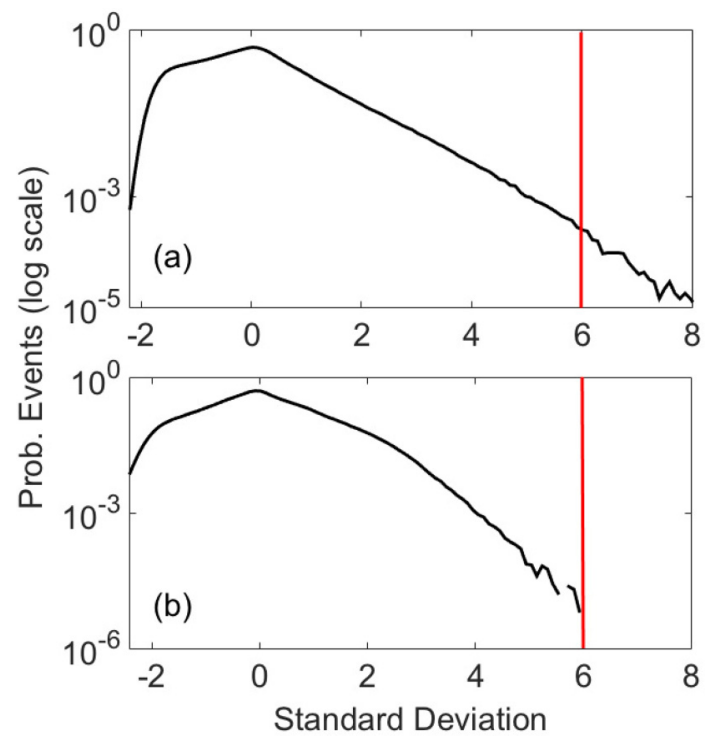

FIG. 2. The standardized pdf's in the (a) LFF and (b) CC regimes. The vertical (solid red) lines at 6 (in units of $\sigma$ ) indicate $E E_{\mathrm{th}}$. The standardized pdf in the LFF regime $(J=9.8 \mathrm{~mA})$ has a long and exponential tail, which leads to a large EE frequency. In the $\mathrm{CC}$ regime $(J=17.4 \mathrm{~mA})$, the pdf has a significantly shorter tail. $L=55 \mathrm{~cm}$ and $\eta=0.6$.

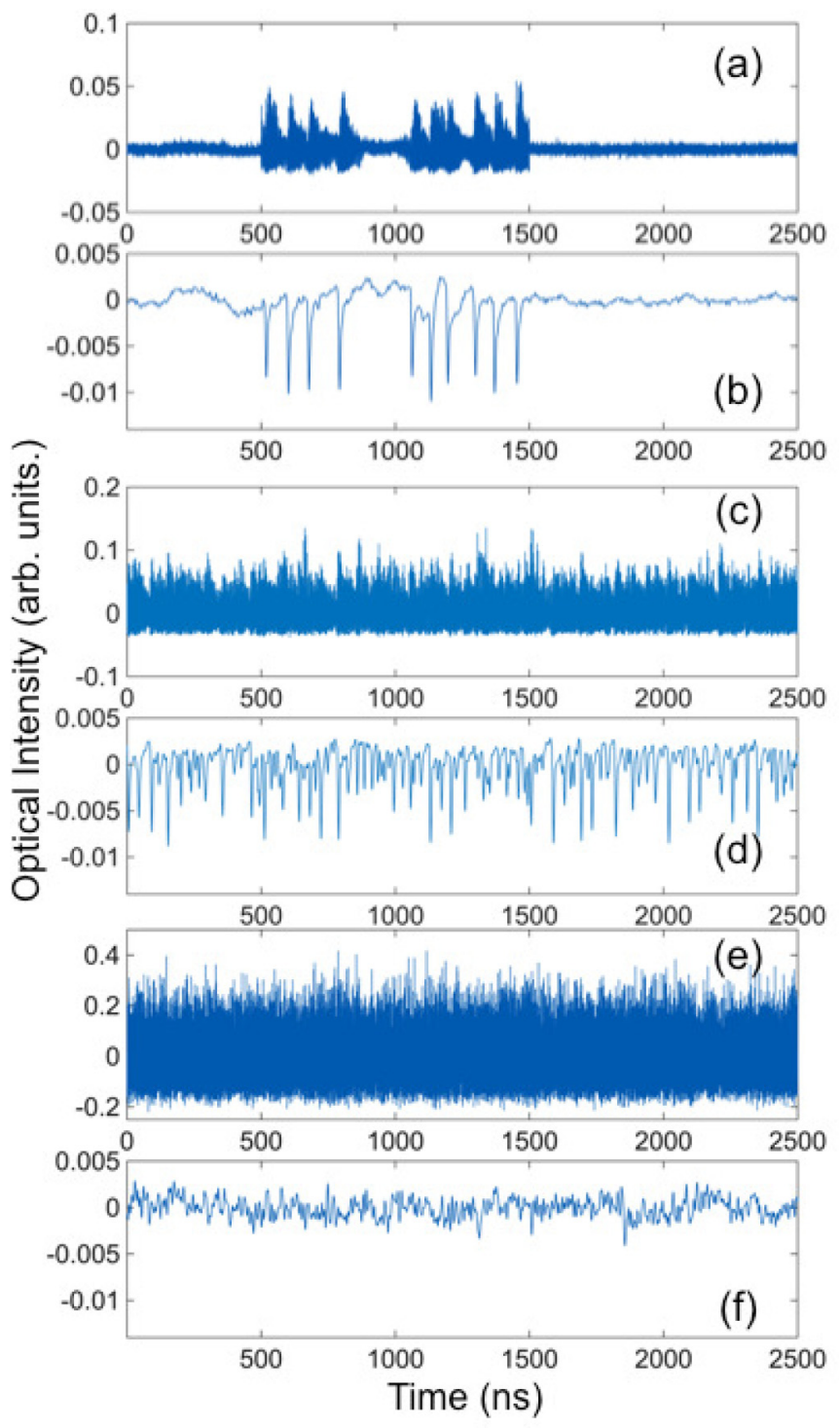

FIG. 3. Examples of the time series $I(t)$ in each dynamical regime: (a),(c),(e) original time series, and (b),(d),(f) time series low-pass filtered at $100 \mathrm{MHz}$. The ECL starts in intermittency (a),(b) and then enters the stable LFF regime (c),(d) in which the intervals between dropouts decrease. The CC regime (e),(f) is recognized by an absence of dropouts. Intermittency is measured at 9.1, stable LFF at 9.8 , and $\mathrm{CC}$ at $17.0 \mathrm{~mA} . L=55 \mathrm{~cm}$ and $\eta=0.75$.

exhibits a significantly long and exponential tail, leading to a large number of EEs. In CC, however, almost no features extend beyond $E E_{\text {th }}$ set by $6 \sigma$; the tail is considerably suppressed by comparison, and the EE frequency negligible. We have also explored the transition between LFF and CC; as $J$ increases and the laser leaves the LFF regime, the tail rapidly becomes shorter. Specifically, for $J=9.8 \mathrm{~mA}$ corresponding to LFF, the probability of observing an EE is $0.18 \%$, a value much larger than one would expect from a Gaussian distribution $\left(\sim 0.2 \times 10^{-6} \%\right)$. Examples of the raw and lowpass filtered $I(t)$ for increasing values of the injection current $J$ are shown in Fig. 3. We have chosen a convenient cutoff frequency to visualize the dropouts easily; all quantitative analysis is carried out on the unfiltered time series. We observe 


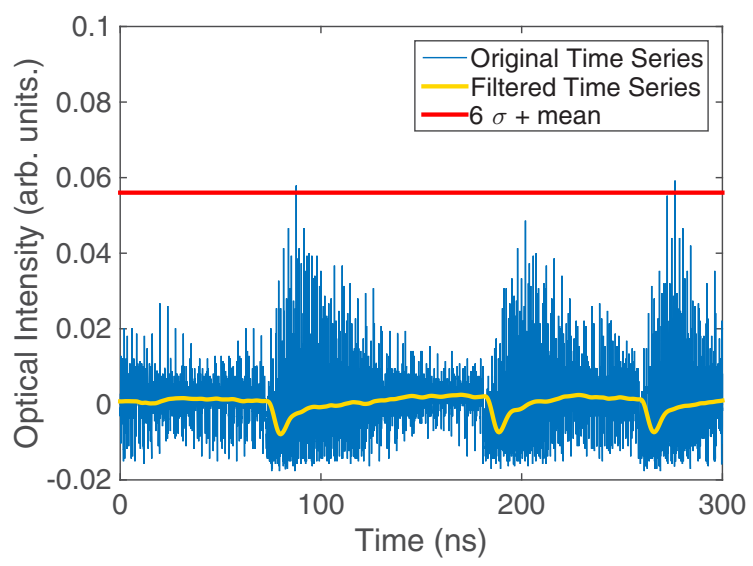

FIG. 4. Large-amplitude ringing in $I(t)$ occurs immediately after dropouts. $J=9.1 \mathrm{~mA}, L=55 \mathrm{~cm}$, and $\eta=0.6$. The red horizontal line shows $E E_{\text {th }}$.

that, for currents close to the threshold, the ECL first enters an intermittent regime, switching between continuous wave (CW) emission, associated with a high-gain mode [22], and transient LFF-like behavior [Figs. 3(a) and 3(b)]. As $J$ is increased, the ECL eventually reaches stable LFF behavior [Figs. 3(c) and 3(d)]. Next, the LFF character of the dynamics is lost as dropouts gradually disappear with increasing current, and the dynamics reaches the fully developed CC regime [Figs. 3(e) and $3(\mathrm{f})]$.

We now show that dropouts are associated with EEs. In Fig. 4, we observe that dropouts are followed by erratic pulses of increasing amplitude. The intensity of these pulses can exceed $E E_{\text {th }}$ and be accounted for as EEs. We can thus infer that EEs are linked to the so-called recovery process after dropouts $[21,23]$. Note though that not all dropouts are followed by EEs.

Figure 5 depicts how the EE frequency, i.e., the number of EEs per unit time, changes over the entire range of the measurement from 8 to $18 \mathrm{~mA}$. After the ECL starts lasing, the intermittent LFF-CW regime appears, in which the EE frequency increases steadily with $J$. It must be noted that in this regime, the calculation of the EEs is based on the LFF periods only. As $J$ is further increased, stable LFF is

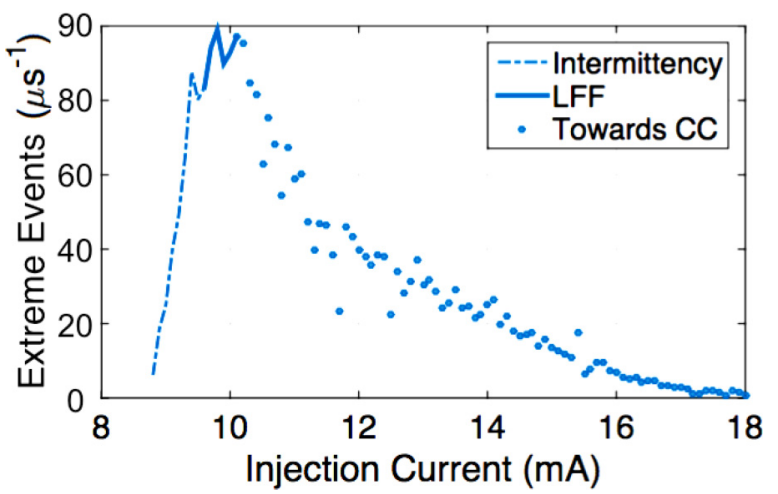

FIG. 5. EE frequency as a function of $J$. EEs are most frequently observed in the LFF regime. As the ECL leaves the stable LFF regime, EE frequency starts to decrease. $L=65 \mathrm{~cm}$ and $\eta=0.75$.

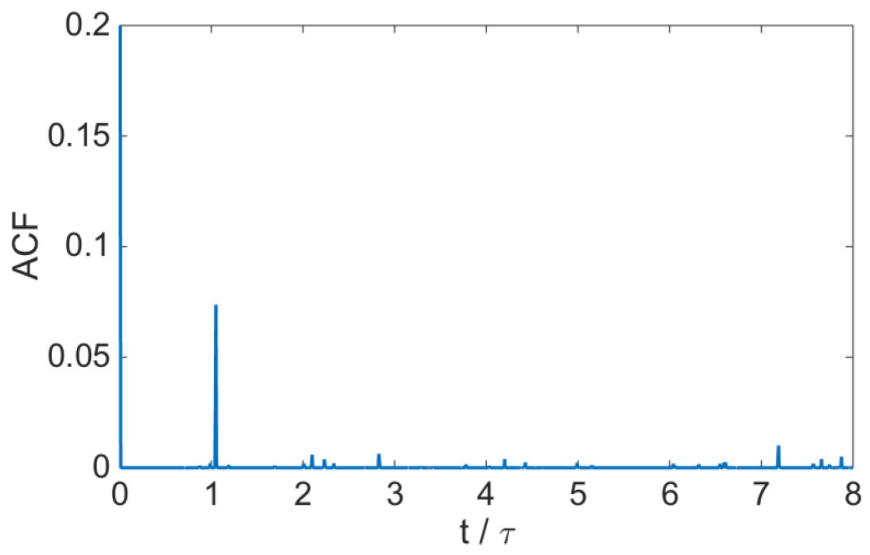

FIG. 6. Normalized autocorrelation function of $I_{E E}(t)$, as defined in the text, in the LFF regime. $\tau$ is the delay time of an external cavity length. We observe a peak around the delay time $\tau(t / \tau=1)$ as well as around $7 \tau$ corresponding to the average time separating consecutive dropouts. $J=10.1 \mathrm{~mA}, L=65 \mathrm{~cm}$, and $\eta=0.75$.

reached, accompanied by the maximum in the EE frequency. For larger values of $J$, the ECL exits the LFF regime and the $\mathrm{EE}$ frequency is seen to steadily decrease. For $J>18 \mathrm{~mA}$, the $\mathrm{EE}$ frequency is negligible. We find that the increase of the $\mathrm{EE}$ frequency before stable LFF is reached is caused by a decrease in the average time between dropouts, in the LFF regime as was also observed in Ref. [24]. As the current is further increased, a transitional regime between LFF and CC [22] is observed, in which dropouts gradually disappear, leading to a progressive decrease in the EE frequency. When the current exceeds $17 \mathrm{~mA}$ approximately, fully developed $\mathrm{CC}$ is observed and the EE frequency goes to zero. Finally, dropouts are no longer seen in $\mathrm{CC}$ resulting in a drastic reduction in the EE frequency.

To better understand temporal correlations between EEs, we explored the autocorrelation function (ACF) of $I_{E E}(t)$ defined as $I_{E E}(t)=I(t)$ if $I(t)>E E_{\text {th }}$ or $I_{E E}(t)=E E_{\text {th }}$ if $I(t) \leqslant E E_{\mathrm{th}}$ as shown in Fig 6. Within the intermittency and

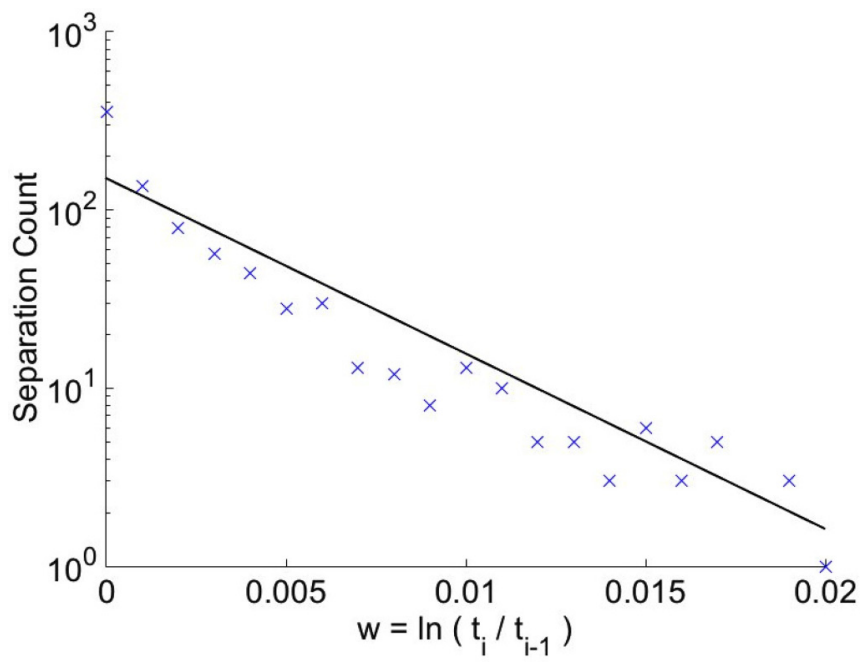

FIG. 7. Statistics of waiting times between successive EEs in the LFF regime. The distribution has log-Poissonian characteristics. $J=$ $9.9 \mathrm{~mA}, L=65 \mathrm{~cm}$, and $\eta=0.75$. 

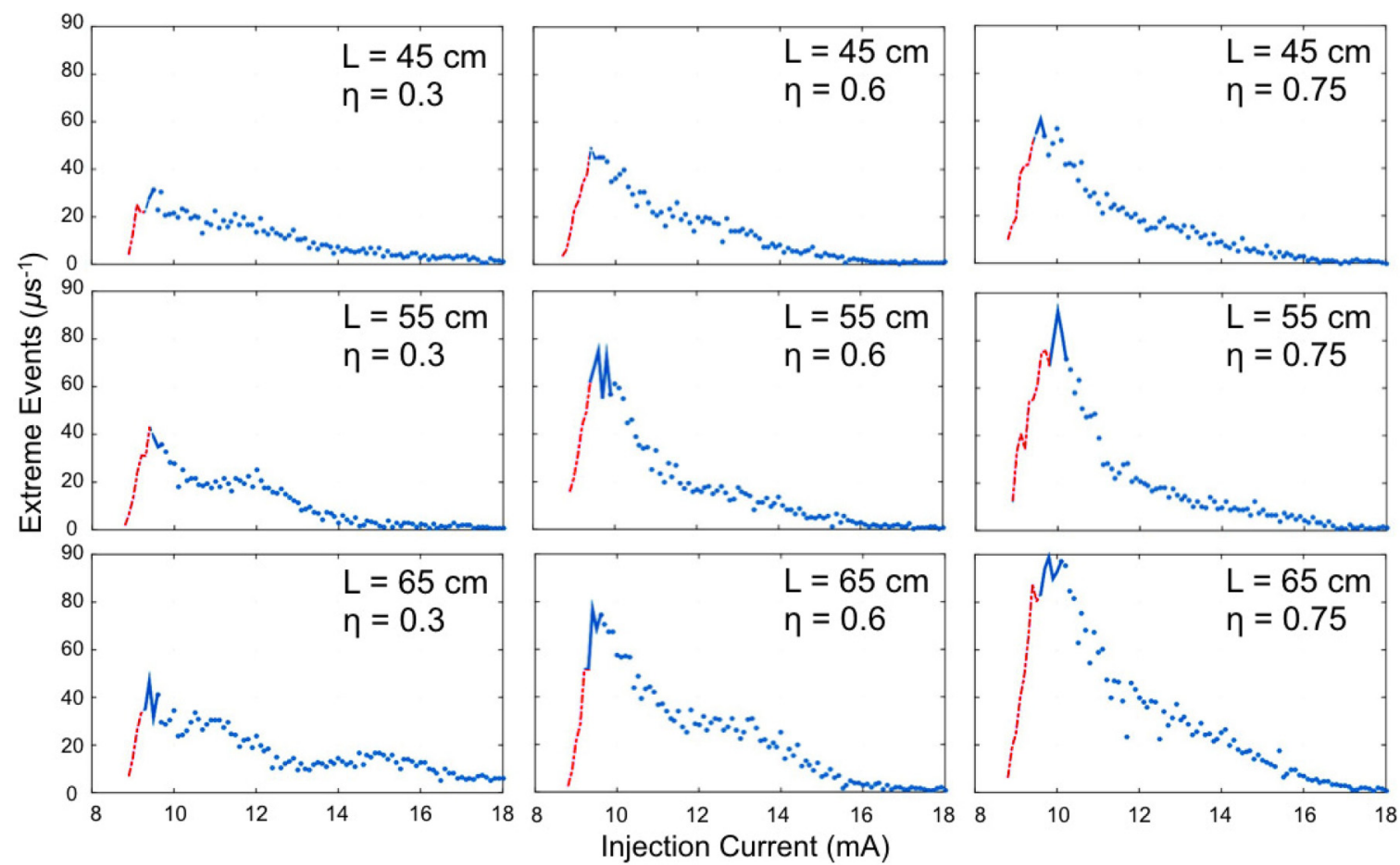

FIG. 8. EE frequency for various values of $L$ and $\eta$ showing that the basic trends as a function of $J$ are essentially robust to changes in these parameters. The maximum EE frequency is observed in all cases to occur in the LFF regimes (blue solid lines). As labeled in Fig 5, the dashed lines (red) and dots indicate the intermittency and CC regimes, respectively.

LFF regimes, the ACF displays a significant peak around the delay time $\tau$ and a peak around the average time in-between dropouts. We find that the peak around $\tau$ corresponds to the fact that, after a dropout, groups of a few consecutive EEs, approximately separated in time by $\tau$, can sometimes be observed, similar to what was reported in Ref. [8] for phase-conjugated feedback. It is interesting to notice that the ACF also exhibits a peak around $7 \tau$, corresponding to the average time between dropouts, and thus confirming the link between the recovery process after a dropout and EEs. Finally, in the transitional regime towards CC, a few EEs can still be observed, but no clear peaks around the delay time or related to the time between dropouts can be identified.

We computed the pdf of the difference between the logarithms of the times of occurrence of successive EEs, i.e., $w=\ln \left(t_{i}\right)-\ln \left(t_{i-1}\right)$ where $t_{i}$ is the time of occurrence of the EE. In the LFF regime, we find a log-Poissonian distribution as shown in Fig. 7. Similarly, a log-Poissonian was found in Refs. $[8,25]$.

We have further investigated the effect of changes in cavity length $L$ and feedback strength $\eta$. Figure 8 illustrates the EE frequency as a function of $J$ for various $L$ and $\eta$. We observe consistently for $L=45,55$, and $65 \mathrm{~cm}$, similar behavior at moderate $\eta=0.3,0.6$, and 0.75: (1) an increase in the EE frequency during intermittency between $\mathrm{CW}$ and LFF, (2) a maximum around stable LFF, and (3) a gradual decrease toward CC. Trends associated with the EE frequency are thus robust with respect to changes in $L$ and $\eta$. In addition, it is noticeable from Fig. 8 that larger feedback levels lead to larger numbers of EEs. We find that this phenomenon is due to an increase of the pulse intensities with increasing feedback.

\section{CONCLUSION}

In summary, we have distinguished in a robust way the LFF and fully developed CC regimes on the basis of extreme events. We have found that while a significant number of EEs are observed when LFF is stable or intermittent, no EEs can be observed in fully developed CC. We attribute this difference to the specific dynamics experienced by the ECL in LFF, which involves an abrupt dropout followed by irregular multi-ps pulses, some of which can qualify as EEs. Interestingly, features similar to those identified numerically as being responsible for EEs in optically injected lasers and for ECLs in the short cavity regime seem to be present in the LFF regimes: the existence of a crisis and of a "narrow door" in phase space. Indeed, according to the Lang and Kobaysashi model, LFF appears in a context of attractor-merging crises that lead to an expansion of the accessible phase space. Moreover, it is known [21] that it is only a portion of the trajectories that approach the maximum gain mode that end up being rejected along an unstable manifold, after going through a "wormhole" in phase space as mentioned in [23], leading thus to a dropout and successively to EEs. We can thus suspect that the existence of a crisis-induced phase space expansion associated with a narrow door for trajectories in phase space is a paradigm for EEs in purely deterministic dynamical systems.

\section{ACKNOWLEDGMENT}

The authors acknowledge support of the Conseil Regional of Lorraine, GT-CNRS-2958, and a 2015 Technologies Incubation scholarship from the Ministry of Education (MOE), Republic of China (Taiwan). 
[1] E. Pelinovsky and C. Kharif, Extreme Ocean Waves (Springer Science \& Business Media, Berlin, 2008).

[2] K. Dysthe, H. E. Krogstad, and P. Müller, Annu. Rev. Fluid Mech. 40, 287 (2008).

[3] D. Sornette, Why Stock Markets Crash: Critical Events in Complex Financial Systems (Princeton University Press, Princeton, 2009).

[4] M. Beniston and D. B. Stephenson, Glob. Planet. Change 44, 1 (2004).

[5] A. N. Ganshin, V. B. Efimov, G. V. Kolmakov, L. P. MezhovDeglin, and P. V. E. McClintock, Phys. Rev. Lett. 101, 065303 (2008).

[6] C. Bonatto, M. Feyereisen, S. Barland, M. Giudici, C. Masoller, J. R. Rios Leite, and J. R. Tredicce, Phys. Rev. Lett. 107, 053901 (2011).

[7] M. G. Kovalsky, A. A. Hnilo, and J. R. Tredicce, Opt. Lett. 36, 4449 (2011).

[8] A. Karsaklian Dal Bosco, D. Wolfersberger, and M. Sciamanna, Opt. Lett. 38, 703 (2013).

[9] J. A. Reinoso, J. Zamora-Munt, and C. Masoller, Phys. Rev. E 87, 062913 (2013).

[10] D. V. Churkin, O. A. Gorbunov, and S. V. Smirnov, Opt. Lett. 36, 3617 (2011).

[11] A. Montina, U. Bortolozzo, S. Residori, and F. T. Arecchi, Phys Rev. Lett. 103, 173901 (2009).

[12] D. Solli, C. Ropers, P. Koonath, and B. Jalali, Nature (London) 450, 1054 (2007).
[13] É. Mercier, A. Even, E. Mirisola, D. Wolfersberger, and M. Sciamanna, Phys. Rev. E 91, 042914 (2015).

[14] S. Randoux and P. Suret, Opt. Lett. 37, 500 (2012).

[15] J. Zamora-Munt, B. Garbin, S. Barland, M. Giudici, J. R. Rios Leite, C. Masoller, and J. R. Tredicce, Phys. Rev. A 87, 035802 (2013).

[16] S. Perrone, R. Vilaseca, J. Zamora-Munt, and C. Masoller, Phys. Rev. A 89, 033804 (2014).

[17] M. C. Soriano, J. García-Ojalvo, C. R. Mirasso, and I. Fischer, Rev. Mod. Phys. 85, 421 (2013).

[18] B. Kim, N. Li, A. Locquet, and D. Citrin, Opt. Express 22, 2348 (2014).

[19] B. Kim, A. Locquet, N. Li, D. Choi, and D. Citrin, IEEE J. Quantum Electron. 50, 965 (2014).

[20] T. Heil, I. Fischer, W. Elsäßer, B. Krauskopf, K. Green, and A. Gavrielides, Phys. Rev. E 67, 066214 (2003).

[21] I. Fischer, G. H. M. van Tartwijk, A. M. Levine, W. Elsässer, E. Göbel, and D. Lenstra, Phys. Rev. Lett. 76, 220 (1996).

[22] T. Heil, I. Fischer, and W. Elsäßer, Phys. Rev. A 58, R2672 (1998).

[23] G. Van Tartwijk, A. Levine, and D. Lenstra, IEEE J. Sel. Top. Quantum Electron. 1, 466 (1995).

[24] A. Hohl, H. J. C. van der Linden, and R. Roy, Opt. Lett. 20, 2396 (1995).

[25] S. Residori, U. Bortolozzo, A. Montina, F. Lenzini, and F. T. Arecchi, Fluct. Noise Lett. 11, 1240014 (2012). 